\title{
Existence of Nontrivial Solutions of $p$-Laplacian Equation with Sign-Changing Weight Functions
}

\author{
Ghanmi Abdeljabbar \\ Département de Mathématiques, Faculté des Sciences de Tunis, Campus Universitaire, 2092 Tunis, Tunisia \\ Correspondence should be addressed to Ghanmi Abdeljabbar; abdeljabbar.ghanmi@lamsin.rnu.tn \\ Received 30 September 2013; Accepted 9 December 2013; Published 12 February 2014 \\ Academic Editors: E. Colorado, L. Gasinski, and D. D. Hai
}

Copyright (C) 2014 Ghanmi Abdeljabbar. This is an open access article distributed under the Creative Commons Attribution License, which permits unrestricted use, distribution, and reproduction in any medium, provided the original work is properly cited.

This paper shows the existence and multiplicity of nontrivial solutions of the $p$-Laplacian problem $-\Delta_{p} u=(1 / \sigma)(\partial F(x, u) / \partial u)+$ $\lambda a(x)|u|^{q-2} u$ for $x \in \Omega$ with zero Dirichlet boundary conditions, where $\Omega$ is a bounded open set in $\mathbb{R}^{n}, 1<q<p<\sigma<p^{*}\left(p^{*}=\right.$ $n p /(n-p)$ if $p<n, p^{*}=\infty$ if $\left.p \geq n\right), \lambda \in \mathbb{R} \backslash\{0\}, a$ is a smooth function which may change sign in $\bar{\Omega}$, and $F \in C^{1}(\bar{\Omega} \times \mathbb{R}, \mathbb{R})$. The method is based on Nehari results on three submanifolds of the space $W_{0}^{1, p}(\Omega)$.

\section{Introduction}

In this paper, we are concerned with the multiplicity of nontrivial nonnegative solutions of the following elliptic equation:

$$
\begin{gathered}
-\Delta_{p} u=\frac{1}{\sigma} \frac{\partial F(x, u)}{\partial u}+\lambda a(x)|u|^{q-2} u \quad \text { in } \Omega, \\
u=0, \quad \text { on } \partial \Omega,
\end{gathered}
$$

where $\Omega$ is a bounded domain of $\mathbb{R}^{n}(n \geq 3), 1<q<p<\sigma<$ $p^{*}\left(p^{*}=n p /(n-p)\right.$ if $p<n, p^{*}=\infty$ if $\left.p \geq n\right), \lambda \in \mathbb{R} \backslash\{0\}, F \in$ $C^{1}(\bar{\Omega} \times \mathbb{R}, \mathbb{R})$ is positively homogeneous of degree $\sigma$; that is, $F(x, t u)=t^{\sigma} F(x, u)$ holds for all $(x, u) \in \bar{\Omega} \times \mathbb{R}$ and the signchanging weight function $a$ satisfies the following condition:

(A) $a \in C(\Omega)$ with $\|a\|_{\infty}=1, a^{+}:=\max (+a, 0) \quad \not \equiv 0$, and $a^{-}:=\max (-a, 0) \not \equiv 0$.

In recent years, several authors have used the Nehari manifold and fibering maps (i.e., maps of the form $t \mapsto$ $J_{\lambda}(t u)$, where $J_{\lambda}$ is the Euler function associated with the equation) to solve semilinear and quasilinear problems. For instance, we cite papers [1-9] and references therein. More precisely, Brown and Zhang [10] studied the following subcritical semilinear elliptic equation with sign-changing weight function:

$$
\begin{gathered}
-\Delta u=\lambda a(x) u+b(x)|u|^{\gamma-2} u(x), \quad \text { in } \Omega, \\
u=0, \quad \text { on } \partial \Omega,
\end{gathered}
$$

where $\gamma>2$. Also, the authors in [10] by the same arguments considered the following semilinear elliptic problem:

$$
\begin{gathered}
-\Delta u=\lambda f(x)|u|^{q-2} u(x)+g(x)|u|^{p-2} u(x), \quad \text { in } \Omega, \\
u=0, \quad \text { on } \partial \Omega,
\end{gathered}
$$

where $1<q<2<p$. Exploiting the relationship between the Nehari manifold and fibering maps, they gave an interesting explanation of the well-known bifurcation result. In fact, the nature of the Nehari manifold changes as the parameter $\lambda$ crosses the bifurcation value.

Inspired by the work of Brown and Zhang [10], Nyamouradi [11] treated the following problem:

$$
\begin{gathered}
\Delta\left(|\Delta u|^{p-2} \Delta u\right)=\frac{1}{p^{*}} f(x, u)+\lambda|u|^{q-2} u(x) ; \quad \text { in } \Omega, \\
u=\Delta u=0, \quad \text { on } \partial \Omega,
\end{gathered}
$$

where $f$ is positively homogeneous of degree $p^{*}-1$. 
In this work, motivated by the above works, we give a very simple variational method to prove the existence of at least two nontrivial solutions of problem (1). In fact, we use the decomposition of the Nehari manifold as $\lambda$ vary to prove our main result.

Before stating our main result, we need the following assumptions:

$\left(\mathrm{H}_{1}\right) F: \bar{\Omega} \times \mathbb{R} \rightarrow \mathbb{R}$ is a $C^{1}$ function such that

$$
F(x, t u)=t^{\sigma} F(x, u)(t>0) \quad \forall x \in \bar{\Omega}, u \in \mathbb{R} .
$$

$\left(\mathrm{H}_{2}\right) F(x, 0)=(\partial F / \partial u)(x, 0)=0, F^{+}(x, u)=$ $\max (+F(x, u), 0) \quad \equiv \quad 0$, and $F^{-}(x, u) \quad=$ $\max (-F(x, u), 0) \not \equiv 0$ for all $u \neq 0$.

We remark that using assumption $\left(\mathrm{H}_{1}\right)$, for all $x \in \bar{\Omega}, u \in \mathbb{R}$, we have the so-called Euler identity:

$$
\begin{gathered}
u \frac{\partial F(x, u)}{\partial u}=\sigma F(x, u), \\
|F(x, u)| \leq K|u|^{\sigma} \text { for some constant } K>0 .
\end{gathered}
$$

Our main result is the following.

Theorem 1. Under the assumptions $(A),\left(H_{1}\right)$, and $\left(H_{2}\right)$, there exists $\lambda_{0}>0$ such that for all $0<|\lambda|<\lambda_{0}$, problem (1) has at least two nontrivial nonnegative solutions.

This paper is organized as follows. In Section 2, we give some notations and preliminaries and we present some technical lemmas which are crucial in the proof of Theorem 1. Theorem 1 is proved in Section 3.

\section{Some Notations and Preliminaries}

Throughout this paper, we denote by $S_{l}$ the best Sobolev constant for the operators $W_{0}^{1, p}(\Omega) \hookrightarrow L^{l}(\Omega)$, given by

$$
S_{l}=\inf _{u \in W_{0}^{1, p}(\Omega) \backslash\{0\}} \frac{\int_{\Omega}|\nabla u|^{p} d x}{\left(\int_{\Omega}|u|^{l}\right)^{p / l} d x},
$$

where $1<l \leq p^{*}$. In particular, we have

$$
\int_{\Omega}|u|^{l} d x \leq S_{l}^{-l / p}\|u\|^{l} \quad \forall u \in W_{0}^{1, p}(\Omega),
$$

with the standard norm

$$
\|u\|=\left(\int_{\Omega}|\nabla u|^{p} d x\right)^{1 / p} .
$$

Problem (1) is posed in the framework of the Sobolev space $E=W_{0}^{1, p}(\Omega)$. Moreover, a function $u$ in $E$ is said to be a weak solution of problem (1) if

$$
\begin{gathered}
\int_{\Omega}|\nabla u|^{p-2} \nabla u \nabla \varphi d x-\frac{1}{\sigma} \int_{\Omega} \frac{\partial F(x, u)}{\partial u} \varphi d x \\
-\lambda \int_{\Omega} a|u|^{q-2} u \varphi d x=0, \quad \forall \varphi \in E .
\end{gathered}
$$

Thus, by (6) the corresponding energy functional of problem (1) is defined in $E$ by

$$
J_{\lambda}(u)=\frac{1}{p}\|u\|^{p}-\frac{1}{\sigma} \int_{\Omega} F(x, u) d x-\frac{\lambda}{q} \int_{\Omega} a(x)|u|^{q} d x .
$$

In order to verify $J_{\lambda} \in C^{1}(E, \mathbb{R})$, we need the following lemmas.

Lemma 2. Assume that $F \in C^{1}(\bar{\Omega} \times \mathbb{R}, \mathbb{R})$ is positively homogeneous of degree $\sigma$; then $\partial F / \partial u \in C(\bar{\Omega} \times \mathbb{R}, \mathbb{R})$ is positively homogeneous of degree $\sigma-1$.

Proof. The proof is the same as that in Chu and Tang [4].

In addition, by Lemma 2, we get the existence of positive constant $M$ such that

$$
\left|\frac{\partial F(x, u)}{\partial u}\right| \leq M|u|^{\sigma-1}, \quad \forall x \in \bar{\Omega}, u \in \mathbb{R} .
$$

Lemma 3 (see [12], Theorem A.2). Let $p, r \in[1, \infty)$ and $f \in$ $C(\bar{\Omega} \times \mathbb{R}, \mathbb{R})$ such that

$$
|f(x, u)| \leq c\left(1+|u|^{p / r}\right), \quad \forall x \in \bar{\Omega}, \forall u \in \mathbb{R} .
$$

Then for every $u \in L^{p}(\Omega)$, one has $f(\cdot, u) \in L^{r}(\Omega)$; moreover the operator $A: L^{p}(\Omega) \rightarrow L^{r}(\Omega)$ defined by $A(u)(x)=$ $f(x, u(x))$ is continuous.

Lemma 4 (See Proposition 1 in [13]). Suppose that $\partial F(x, u) / \partial u \in C(\bar{\Omega} \times \mathbb{R}, \mathbb{R})$ verifies condition (12). Then, the functional $J_{\lambda}$ belongs to $C^{1}(E, \mathbb{R})$, and

$$
\left\langle J_{\lambda}^{\prime}(u), u\right\rangle=\|u\|^{p}-\int_{\Omega} F(x, u) d x-\lambda \int_{\Omega} a(x)|u|^{q} d x,
$$

where $\langle\cdot, \cdot\rangle$ denotes the usual duality between $E$ and $E^{*}:=$ $W^{-1, p^{\prime}}(\Omega)$ (the dual space of the sobolev space $E$ ).

As the energy functional $J_{\lambda}$ is not bounded below in $E$, it is useful to consider the functional on the Nehari manifold:

$$
N_{\lambda}=\left\{u \in E \backslash\{0\}:\left\langle J_{\lambda}^{\prime}(u), u\right\rangle=0\right\} .
$$

Thus, $u \in N_{\lambda}$ if and only if

$$
\|u\|^{p}-\int_{\Omega} F(x, u) d x-\lambda \int_{\Omega} a(x)|u|^{q} d x=0 .
$$

Note that $N_{\lambda}$ contains every nonzero solution of problem (1). Moreover, one has the following result.

Lemma 5. The energy functional $J_{\lambda}$ is coercive and bounded below on $N_{\lambda}$.

Proof. If $u \in N_{\lambda}$, then by (16) and condition (A) we obtain

$$
\begin{aligned}
J_{\lambda}(u) & =\frac{\sigma-p}{\sigma p}\|u\|^{p}-\lambda \frac{\sigma-q}{\sigma q} \int_{\Omega} a(x)|u|^{q} d x, \\
& \geq \frac{\sigma-p}{\sigma p}\|u\|^{p}-|\lambda| \frac{\sigma-q}{\sigma q} \int_{\Omega}|u|^{q} d x .
\end{aligned}
$$


So, it follows from (8) that

$$
J_{\lambda}(u) \geq \frac{\sigma-p}{\sigma p}\|u\|^{p}-\frac{|\lambda|}{S_{q}^{q / p}} \frac{\sigma-q}{\sigma q}\|u\|^{q} .
$$

Thus, $J_{\lambda}$ is coercive and bounded below on $N_{\lambda}$.

Define

$$
\phi_{\lambda}(u)=\left\langle J_{\lambda}^{\prime}(u), u\right\rangle
$$

Then, by (16) it is easy to see that for $u \in N_{\lambda}$,

$$
\begin{aligned}
& \left\langle\phi_{\lambda}^{\prime}(u), u\right\rangle \\
& =p\|u\|^{p}-\sigma \int_{\Omega} F(x, u) d x-\lambda q \int_{\Omega} a(x)|u|^{q} d x \\
& =\lambda(p-q) \int_{\Omega} a(x)|u|^{q} d x-(\sigma-p) \int_{\Omega} F(x, u) d x \\
& =\lambda(\sigma-q) \int_{\Omega} a(x)|u|^{q} d x-(\sigma-p)\|u\|^{p} \\
& =(p-q)\|u\|^{p}-(\sigma-q) \int_{\Omega} F(x, u) d x
\end{aligned}
$$

Now, we split $N_{\lambda}$ into three parts

$$
\begin{aligned}
& N_{\lambda}^{+}=\left\{u \in N_{\lambda}:\left\langle\phi_{\lambda}^{\prime}(u), u\right\rangle>0\right\}, \\
& N_{\lambda}^{0}=\left\{u \in N_{\lambda}:\left\langle\phi_{\lambda}^{\prime}(u), u\right\rangle=0\right\}, \\
& N_{\lambda}^{-}=\left\{u \in N_{\lambda}:\left\langle\phi_{\lambda}^{\prime}(u), u\right\rangle<0\right\} .
\end{aligned}
$$

Lemma 6. Assume that $u_{0}$ is a local minimizer for $J_{\lambda}$ on $N_{\lambda}$ and that $u_{0} \notin N_{\lambda}^{0}$. Then, $J_{\lambda}^{\prime}\left(u_{0}\right)=0$ in $E^{-1}$ (the dual space of the Sobolev space E).

Proof. Our proof is the same as that in Brown-Zhang [10, Theorem 2.3].

Lemma 7. One has the following:

(i) if $u \in N_{\lambda}^{+}$, then $\lambda \int_{\Omega} a(x)|u|^{q} d x>0$;

(ii) if $u \in N_{\lambda}^{0}$, then $\lambda \int_{\Omega} a(x)|u|^{q} d x>0$ and $\int_{\Omega} F(x, u) d x>0$;

(iii) if $u \in N_{\lambda}^{-}$, then $\int_{\Omega} F(x, u) d x>0$.

Proof. The proof is immediate from (21), (22), and (23).

From now on, we denote by $\lambda_{0}$ the constant defined by

$$
\lambda_{0}=\frac{q(\sigma-p)}{p(\sigma-q)} S_{q}^{q / p}\left(\frac{p-q}{K(\sigma-q)} S_{\sigma}^{\sigma / p}\right)^{(p-q)(\sigma-p)},
$$

then we have the following.

Lemma 8. If $0<|\lambda|<\lambda_{0}$, then $N_{\lambda}^{0}=\varnothing$.
Proof. Suppose otherwise, that $0<|\lambda|<\lambda_{0}$ such that $N_{\lambda}^{0} \neq \varnothing$. Then for $u \in N_{\lambda}^{0}$, we have

$$
0=\left\langle\phi_{\lambda}^{\prime}(u), u\right\rangle=\lambda(\sigma-q) \int_{\Omega} a(x)|u|^{q} d x-(\sigma-p)\|u\|^{p}
$$

$$
=(p-q)\|u\|^{p}-(\sigma-q) \int_{\Omega} F(x, u) d x .
$$

From the Hölder inequality, (6) and (8), it follows that

$$
\begin{aligned}
\int_{\Omega} F(x, u) d x & \leq \int_{\Omega}|F(x, u)| d x \\
& \leq K \int_{\Omega}|u|^{\sigma} d x \leq K S_{\sigma}^{-\sigma / p}\|u\|^{\sigma}
\end{aligned}
$$

Hence, it follows from (27) that

$$
\|u\|^{p}=\frac{\sigma-q}{p-q} \int_{\Omega} F(x, u) d x \leq \frac{\sigma-q}{p-q} K S_{\sigma}^{-\sigma / p}\|u\|^{\sigma},
$$

then,

$$
\|u\| \geq\left(\frac{p-q}{K(\sigma-q)} S_{\sigma}^{\sigma / p}\right)^{1 /(\sigma-p)}
$$

On the other hand, from condition (A), (8) and (26) we have

$$
\|u\|^{p}=\lambda \frac{\sigma-q}{\sigma-p} \int_{\Omega} a(x)|u|^{q} d x \leq|\lambda| \frac{\sigma-q}{\sigma-p} S_{q}^{-q / p}\|u\|^{q} .
$$

So,

$$
\|u\| \leq\left(|\lambda| \frac{\sigma-q}{\sigma-p} S_{q}^{-q / p}\right)^{1 /(p-q)} .
$$

Combining (30) and (32), we obtain $\lambda_{0} \leq|\lambda|$, which is a contradiction.

By Lemma 8, for $0<|\lambda|<\lambda_{0}$, we write $N_{\lambda}=N_{\lambda}^{+} \cup N_{\lambda}^{-}$ and define

$$
\theta_{\lambda}=\inf _{u \in N_{\lambda}} J_{\lambda}(u), \quad \theta_{\lambda}^{+}=\inf _{u \in N_{\lambda}^{+}} J_{\lambda}(u), \quad \theta_{\lambda}^{-}=\inf _{u \in N_{\lambda}^{-}} J_{\lambda}(u) .
$$

Then, we have the following.

Lemma 9. If $0<|\lambda|<\lambda_{0}$, then

$$
\theta_{\lambda} \leq \theta_{\lambda}^{+}<0, \quad \theta_{\lambda}^{-}>d_{0}
$$

for some $d_{0}>0$ depending on $p, q, \sigma, K, \lambda, S_{q}$, and $S_{\sigma}$.

Proof. Let $u \in N_{\lambda}^{+}$. Then, from (23) we have

$$
\frac{p-q}{\sigma-q}\|u\|^{p}>\int_{\Omega} F(x, u) d x .
$$


So

$$
\begin{aligned}
J_{\lambda}(u) & =\frac{q-p}{p q}\|u\|^{p}+\frac{\sigma-q}{\sigma q} \int_{\Omega} F(x, u) d x \\
& <\left(\frac{q-p}{p q}+\frac{\sigma-q}{\sigma q} \frac{p-q}{\sigma-q}\right)\|u\|^{p} \\
& =-\frac{(p-q)(\sigma-p)}{p q \sigma}\|u\|^{p}<0 .
\end{aligned}
$$

Thus, from the definition of $\theta_{\lambda}$ and $\theta_{\lambda}^{+}$, we can deduce that $\theta_{\lambda} \leq \theta_{\lambda}^{+}<0$.

Now, let $u \in N_{\lambda}^{-}$. Then, using (6) and (8) we obtain

$$
\frac{p-q}{\sigma-q}\|u\|^{p}<\int_{\Omega} F(x, u) d x \leq K S_{\sigma}^{-\sigma / p}\|u\|^{\sigma},
$$

this implies that

$$
\|u\|>\left(\frac{p-q}{\sigma-q} \frac{S_{\sigma}^{\sigma / p}}{K}\right)^{1 /(\sigma-p)}, \quad \forall u \in N_{\lambda}^{-} .
$$

In addition, by (18) and (38)

$$
\begin{aligned}
J_{\lambda}(u) & \geq \frac{\sigma-p}{p \sigma}\|u\|^{p}-|\lambda| S_{q}^{-q / p} \frac{\sigma-p}{\sigma q}\|u\|^{q} \\
& \geq\|u\|^{q}\left[\frac{\sigma-p}{p \sigma}\|u\|^{p-q}-|\lambda| S_{q}^{-q / p} \frac{\sigma-q}{\sigma q}\right] \\
& >\left(\frac{p-q}{\sigma-q} \frac{S_{\sigma}^{\sigma / p}}{K}\right)^{q /(\sigma-p)}\left(\frac{\sigma-p}{p \sigma}\left(\frac{p-q}{\sigma-q} \frac{S_{\sigma}^{\sigma / p}}{K}\right)^{(p-q) /(\sigma-p)}\right. \\
-|\lambda| S_{q}^{-q / p} \frac{\sigma-q}{\sigma q} &
\end{aligned}
$$

Thus, since $0<|\lambda|<\lambda_{0}$, we conclude that $J_{\lambda}>d_{0}$ for some $d_{0}>0$. This completes the proof.

For $u \in E$ with $\int_{\Omega} F(x, u) d x>0$, set

$$
T=\left(\frac{(p-q)\|u\|^{p}}{(\sigma-q) \int_{\Omega} F(x, u) d x}\right)^{1 /(\sigma-p)}>0 .
$$

Then, the following lemma holds.

Lemma 10. For each $u \in E$ with $\int_{\Omega} F(x, u) d x>0$, one has the following:

(i) if $\lambda \int_{\Omega} a(x)|u|^{q} d x \leq 0$, then there exists unique $t^{-}>T$ such that $t^{-} u \in N_{\lambda}^{-}$and

$$
J_{\lambda}\left(t^{-} u\right)=\sup _{t \geq 0} J_{\lambda}(t u)
$$

(ii) if $\lambda \int_{\Omega} a(x)|u|^{q} d x>0$, then there are unique $0<t^{+}<$ $T<t^{-}$such that $\left(t^{-} u, t^{+} u\right) \in N_{\lambda}^{-} \times N_{\lambda}^{+}$and

$$
J_{\lambda}\left(t^{-} u\right)=\sup _{t \geq 0} J_{\lambda}(t u), \quad J_{\lambda}\left(t^{+} u\right)=\inf _{0 \leq t \leq T} J_{\lambda}(t u) .
$$

Proof. We fix $u \in E$ with $\int_{\Omega} F(x, u) d x>0$ and we let

$$
m(t)=t^{p-q}\|u\|^{p}-t^{\sigma-q} \int_{\Omega} F(x, u) d x \quad \text { for } t \geq 0 .
$$

Then, it is easy to check that $m(t)$ achieves its maximum at $T$. Moreover,

$$
\begin{aligned}
m(T)= & \|u\|^{q}\left[\left(\frac{p-q}{\sigma-q}\right)^{(p-q)(\sigma-p)}-\left(\frac{p-q}{\sigma-q}\right)^{(\sigma-q) /(\sigma-p)}\right] \\
& \times\left(\frac{\|u\|^{\sigma}}{\int_{\Omega} F(x, u) d x}\right)^{(p-q) /(\sigma-p)} \\
\geq & \|u\|^{q}\left(\frac{\sigma-p}{\sigma-q}\right)\left(\frac{(\sigma-q) S_{\sigma}^{\sigma / p}}{K(p-q)}\right)^{(p-q) /(\sigma-p)} .
\end{aligned}
$$

(i) We suppose that $\lambda \int_{\Omega} a(x)|u|^{q} d x \leq 0$. Since $m(0)=$ $0, m(t) \rightarrow-\infty$ as $t \rightarrow \infty, m^{\prime}(t)>0$ for $t<T$ and $m^{\prime}(t)<$ 0 for $t>T$. There is a unique $t^{-}>T$ such that $m\left(t^{-}\right)=$ $\lambda \int_{\Omega} a(x)|u|^{q} d x \leq 0$.

Now, it follows from (14) and (27) that

$$
\begin{gathered}
\phi_{\lambda}^{\prime}\left(t^{-} u\right) t^{-} u=\left(t^{-}\right)^{1+q} m^{\prime}\left(t^{-}\right)<0, \\
J_{\lambda}^{\prime}\left(t^{-} u\right) t^{-} u=\left(t^{-}\right)^{q}\left(m\left(t^{-}\right)-\lambda \int_{\Omega} a(x)|u|^{q} d x\right)=0 .
\end{gathered}
$$

Hence, $t^{-} u \in N_{\lambda}^{-}$. On the other hand, it is easy to see that for all $t>T$

$$
\frac{d^{2}}{d t^{2}} J_{\lambda}(t u)<0, \quad \frac{d}{d t} J_{\lambda}(t u)=0 \quad \text { for } t=t^{-}
$$

Thus, $J_{\lambda}\left(t^{-} u\right)=\sup _{t \geq 0} J_{\lambda}(t u)$.

(ii) We suppose that $\lambda \int_{\Omega} a(x)|u|^{q} d x>0$. Then, by (A), (8) and the fact that $|\lambda|<\lambda_{0}$ we obtain

$$
m(0)=0<\lambda \int_{\Omega} a(x)|u|^{q} d x \leq|\lambda| S_{q}^{-q / p}\|u\|^{q}<m(T) .
$$

Then, there are unique $t^{+}$and $t^{-}$such that $0<t^{+}<T<t^{-}$, $m\left(t^{+}\right)=\lambda \int_{\Omega} a(x)|u|^{q} d x=m\left(t^{-}\right)$, and $m^{\prime}\left(t^{-}\right)<0<m^{\prime}\left(t^{+}\right)$. We have $\left(t^{-} u, t^{+} u\right) \in N_{\lambda}^{-} \times N_{\lambda}^{+}$, and

$$
\begin{gathered}
J_{\lambda}\left(t^{+} u\right) \leq J_{\lambda}(t u) \leq J_{\lambda}\left(t^{-} u\right) \quad \forall t \in\left[t^{+}, t^{-}\right], \\
J_{\lambda}\left(t^{+} u\right) \leq J_{\lambda}(t u) \quad \forall 0 \leq t \leq t^{+} .
\end{gathered}
$$

Thus,

$$
J_{\lambda}\left(t^{-} u\right)=\sup _{t \geq 0} J_{\lambda}(t u), \quad J_{\lambda}\left(t^{+} u\right)=\inf _{0 \leq t \leq T} J_{\lambda}(t u) .
$$

This completes the proof. 
For each $u \in E$ with $\lambda \int_{\Omega} a(x)|u|^{q} d x>0$, set

$$
\widetilde{T}=\left(\frac{(\sigma-q) \lambda \int_{\Omega} a(x)|u|^{q} d x}{(\sigma-p)\|u\|^{p}}\right)^{1 /(p-q)}>0
$$

Then we have the following.

Lemma 11. For each $u \in E$ with $\lambda \int_{\Omega} a(x)|u|^{q} d x>0$, one has the following:

(i) if $\int_{\Omega} F(x, u) d x \leq 0$, then there exists a unique $0<t^{+}<$ $\widetilde{T}$ such that $t^{+} u \in N_{\lambda}^{+}$and

$$
J_{\lambda}\left(t^{+} u\right)=\inf _{t \geq 0} J_{\lambda}(t u)
$$

(ii) if $\int_{\Omega} F(x, u) d x>0$, then there are unique $0<t^{+}<$ $\widetilde{T}<t^{-}$such that $\left(t^{-} u, t^{+} u\right) \in N_{\lambda}^{-} \times N_{\lambda}^{+}$and

$$
J_{\lambda}\left(t^{-} u\right)=\sup _{t \geq 0} J_{\lambda}(t u), \quad J_{\lambda}\left(t^{+} u\right)=\inf _{0 \leq t \leq \widetilde{T}} J_{\lambda}(t u) .
$$

Proof. For $u \in E$ with $\lambda \int_{\Omega} a(x)|u|^{q} d x>0$, we can take

$$
\widetilde{m}(t)=t^{p-\sigma}\|u\|^{p}-\lambda t^{q-\sigma} \int_{\Omega} a(x)|u|^{q} d x \quad \text { for } t>0
$$

and similar to the argument in Lemma 9, we obtain the results of Lemma 10.

Proposition 12. (i) There exist minimizing sequences $\left\{u_{n}^{+}\right\}$in $N_{\lambda}^{+}$such that

$$
J_{\lambda}\left(u_{n}^{+}\right)=\theta_{\lambda}^{+}+\circ(1), \quad J_{\lambda}^{\prime}\left(u_{n}^{+}\right)=\circ(1) \quad \text { in } E^{-1} .
$$

(ii) There exist minimizing sequences $\left\{u_{n}^{-}\right\}$in $N_{\lambda}^{-}$such that

$$
J_{\lambda}\left(u_{n}^{-}\right)=\theta_{\lambda}^{-}+\circ(1), \quad J_{\lambda}^{\prime}\left(u_{n}^{-}\right)=\circ(1) \quad \text { in } E^{-1} .
$$

Proof. The proof is almost the same as that in Wu [14, Proposition 9] and is omitted here.

\section{Proof of Our Result}

Throughout this section, the norm $L^{s}$ is denoted by $\|\cdot\|_{s}$ for $1 \leq s \leq \infty$ and the parameter $\lambda$ satisfies $0<|\lambda|<\lambda_{0}$.

Theorem 13. If $0<|\lambda|<\lambda_{0}$, then, problem (1) has a positive solution $u_{0}^{+}$in $N_{\lambda}^{+}$such that

$$
J_{\lambda}\left(u_{0}^{+}\right)=\theta_{\lambda}=\theta_{\lambda}^{+}
$$

Proof. By Proposition 12(i), there exists a minimizing sequence $\left\{u_{n}^{+}\right\}$for $J_{\lambda}$ on $N_{\lambda}^{+}$such that

$$
J_{\lambda}\left(u_{n}^{+}\right)=\theta_{\lambda}^{+}+o(1), \quad J_{\lambda}^{\prime}\left(u_{n}^{+}\right)=o(1) \quad \text { in } E^{-1} .
$$

Then by Lemma 5 , there exists a subsequence $\left\{u_{n}\right\}$ and $u_{0}^{+}$in $E$ such that

$$
\begin{gathered}
u_{n} \rightarrow u_{0}^{+} \quad \text { weakly in } E, \\
u_{n} \longrightarrow u_{0}^{+} \quad \text { strongly in } L^{q}(\Omega) \text { and in } L^{\sigma}(\Omega) .
\end{gathered}
$$

This implies that $\int_{\Omega} a(x)\left|u_{n}\right|^{q} d x \rightarrow \int_{\Omega} a(x)\left|u_{0}^{+}\right|^{q} d x$ as $n \rightarrow$ $\infty$.

Next, we will show that

$$
\int_{\Omega} F\left(x, u_{n}\right) d x \longrightarrow \int_{\Omega} F\left(x, u_{0}^{+}\right) d x \quad \text { as } n \longrightarrow \infty \text {. }
$$

By Lemma 3, we have

$$
\frac{\partial F\left(x, u_{n}\right)}{\partial u} \in L^{\gamma}(\Omega), \quad \frac{\partial F\left(x, u_{n}\right)}{\partial u} \longrightarrow \frac{\partial F\left(x, u_{0}^{+}\right)}{\partial u} \quad \text { in } L^{\gamma}(\Omega),
$$

where $\gamma=\sigma /(\sigma-1)$. On the other hand, it follows from the Hölder inequality that

$$
\begin{gathered}
\int_{\Omega}\left|u_{n} \frac{\partial F\left(x, u_{n}\right)}{\partial u}-u_{0}^{+} \frac{\partial F\left(x, u_{0}^{+}\right)}{\partial u}\right| d x \\
\leq \int_{\Omega}\left|\left(u_{n}-u_{0}^{+}\right) \frac{\partial F\left(x, u_{n}\right)}{\partial u}\right| d x \\
\quad+\int_{\Omega}\left|u_{0}^{+}\right|\left|\frac{\partial F\left(x, u_{n}\right)}{\partial u}-\frac{\partial F\left(x, u_{0}^{+}\right)}{\partial u}\right| d x \\
\leq\left\|u_{n}-u_{0}^{+}\right\|_{\sigma}\left\|\frac{\partial F\left(x, u_{n}\right)}{\partial u}\right\|_{\gamma} \\
\quad+\left\|u_{0}^{+}\right\|_{\sigma}\left\|\frac{\partial F\left(x, u_{n}\right)}{\partial u}-\frac{\partial F\left(x, u_{0}^{+}\right)}{\partial u}\right\|_{\gamma} \\
\longrightarrow 0 \text { as } n \longrightarrow \infty .
\end{gathered}
$$

Hence, $\int_{\Omega} F\left(x, u_{n}\right) d x \rightarrow \int_{\Omega} F\left(x, u_{0}^{+}\right) d x$ as $n \rightarrow \infty$.

By (57) and (58) it is easy to prove that $u_{0}^{+}$is a weak solution of (1).

Since

$$
\begin{aligned}
J_{\lambda}\left(u_{n}\right) & =\frac{\sigma-p}{p \sigma}\left\|u_{n}\right\|^{p}-\lambda \frac{\sigma-q}{q \sigma} \int_{\Omega} a(x)\left|u_{n}\right|^{q} d x \\
& \geq-\lambda \frac{\sigma-q}{q \sigma} \int_{\Omega} a(x)\left|u_{n}\right|^{q} d x
\end{aligned}
$$

then by (57) and Lemma 9, we have $J_{\lambda}\left(u_{n}\right) \rightarrow \theta_{\lambda}<0$ as $n \rightarrow \infty$. Letting $n \rightarrow \infty$, we obtain

$$
\lambda \int_{\Omega} a(x)\left|u_{0}^{+}\right|^{q} d x>0
$$

Now, we aim to prove that $u_{n} \rightarrow u_{0}^{+}$strongly in $E$ and $J_{\lambda}\left(u_{0}^{+}\right)=\theta_{\lambda}$ 
Using the fact that $u_{0}^{+} \in N_{\lambda}$ and by Fatou's lemma, we get

$$
\begin{gathered}
\theta_{\lambda} \leq J_{\lambda}\left(u_{0}^{+}\right)=\frac{1}{p}\left\|u_{0}^{+}\right\|^{p}-\frac{1}{\sigma} \int_{\Omega} F\left(x, u_{0}^{+}\right) d x \\
-\frac{\lambda}{q} \int_{\Omega} a(x)\left|u_{0}^{+}\right|^{q} d x \\
\leq \liminf _{n \rightarrow \infty}\left(\frac{1}{p}\left\|u_{n}\right\|^{p}-\frac{1}{\sigma} \int_{\Omega} F\left(x, u_{n}\right) d x\right. \\
\left.-\frac{\lambda}{q} \int_{\Omega} a(x)\left|u_{n}\right|^{q} d x\right) \\
\leq \liminf _{n \rightarrow \infty} J_{\lambda}\left(u_{n}\right)=\theta_{\lambda} .
\end{gathered}
$$

This implies that

$$
J_{\lambda}\left(u_{0}^{+}\right)=\theta_{\lambda}, \quad \lim _{n \rightarrow \infty}\left\|u_{n}\right\|^{p}=\left\|u_{0}^{+}\right\|^{p} .
$$

Let $\widetilde{u}_{n}=u_{n}-u_{0}^{+}$; then by Brézis-Lieb Lemma [3] we obtain

$$
\lim _{n \rightarrow \infty}\left(\left\|u_{n}\right\|^{p}-\left\|\widetilde{u}_{n}\right\|^{p}\right)=\left\|u_{0}^{+}\right\|^{p} .
$$

Therefore, $u_{n} \rightarrow u_{0}^{+}$strongly in $E$.

Moreover, we have $u_{0}^{+} \in N_{\lambda}^{+}$. In fact, if $u_{0}^{+} \in N_{\lambda}^{-}$then, there exist $t_{0}^{+}$, $t_{0}^{-}$such that $t_{0}^{-} u_{0}^{+} \in N_{\lambda}^{-}$and $t_{0}^{+} u_{0}^{+} \in N_{\lambda}^{+}$. In particular we have $t_{0}^{+}<t_{0}^{-}=1$. Since

$$
\frac{d^{2}}{d t^{2}} J_{\lambda}\left(t_{0}^{+} u_{0}^{+}\right)>0, \quad \frac{d}{d t} J_{\lambda}\left(t_{0}^{+} u_{0}^{+}\right)=0,
$$

there exists $t_{0}^{+}<\widetilde{t}<t_{0}^{-}$such that $J_{\lambda}\left(t_{0}^{+} u_{0}^{+}\right)<J_{\lambda}\left(\widetilde{t} u_{0}^{+}\right)$. By Lemma 10, we have

$$
J_{\lambda}\left(t_{0}^{+} u_{0}^{+}\right)<J_{\lambda}\left(\widetilde{t} u_{0}^{+}\right) \leq J_{\lambda}\left(t_{0}^{-} u_{0}^{+}\right)=J_{\lambda}\left(u_{0}^{+}\right),
$$

which is a contradiction.

Finally, by (63) we may assume that $u_{0}^{+}$is a nontrivial nonnegative solution of problem (1).

Theorem 14. If $0<|\lambda|<\lambda_{0}$, then, problem (1) has a positive solution $u_{0}^{-}$in $N_{\lambda}^{-}$such that

$$
J_{\lambda}\left(u_{0}^{-}\right)=\theta_{\lambda}^{-} .
$$

Proof. By Proposition 12(ii), there exists a minimizing sequence $\left\{u_{n}\right\}$ for $J_{\lambda}$ on $N_{\lambda}^{-}$such that

$$
\begin{aligned}
& J_{\lambda}\left(u_{n}\right)=\theta_{\lambda}^{-}+o(1), \quad J_{\lambda}^{\prime}\left(u_{n}\right)=o(1) \text { in } E^{-1}, \\
& u_{n} \rightarrow u_{0}^{-} \quad \text { weakly in } E, \\
& u_{n} \longrightarrow u_{0}^{-} \quad \text { strongly in } L^{q}(\Omega) \text { and in } L^{\sigma}(\Omega) .
\end{aligned}
$$

Moreover, by (23) we obtain

$$
\int_{\Omega} F\left(x, u_{n}\right) d x>\frac{p-q}{\sigma-q}\left\|u_{n}\right\|^{p} .
$$

So, by (38) and (72) there exists a positive constant $\widetilde{C}$ such that

$$
\int_{\Omega} F\left(x, u_{n}\right)>\widetilde{C}
$$

This implies that

$$
\int_{\Omega} F\left(x, u_{0}^{-}\right) \geq \widetilde{C} .
$$

By (70) and (71), we obtain clearly that $u_{0}^{-}$is a weak solution of (1).

Now, we aim to prove that $u_{n} \rightarrow u_{0}^{-}$strongly in $E$. Supposing otherwise, then

$$
\left\|u_{0}^{-}\right\|<\liminf _{n \rightarrow \infty}\left\|u_{n}\right\| .
$$

By Lemma 9 , there is a unique $t_{0}^{-}$such that $t_{0}^{-} u_{0}^{-} \in N_{\lambda}^{-}$. Since $u_{n} \in N_{\lambda}^{-}, J_{\lambda}\left(u_{n}\right) \geq J_{\lambda}\left(t u_{n}\right)$ for all $t \geq 0$, we have

$$
J_{\lambda}\left(t_{0}^{-} u_{0}^{-}\right)<\lim _{n \rightarrow \infty} J_{\lambda}\left(t_{0}^{-} u_{n}\right) \leq \lim _{n \rightarrow \infty} J_{\lambda}\left(u_{n}\right)=\theta_{\lambda}^{-},
$$

which is a contradiction. Hence $u_{n} \rightarrow u_{0}^{-}$strongly in $E$.

This imply that

$$
J_{\lambda}\left(u_{n}\right) \longrightarrow J_{\lambda}\left(u_{0}^{-}\right)=\theta_{\lambda}^{-} \text {as } n \longrightarrow \infty .
$$

By Lemma 5 and (74) we may assume that $u_{0}^{-}$is a nontrivial solution of problem (1).

Now, we begin to show the proof of Theorem 1: by Theorem 13, we obtain that for all $0<\lambda<\lambda_{0}$, problem (1) has a nontrivial solution $u_{0}^{+} \in N_{\lambda}^{+}$. On the other hand, from Theorem 14, we get the second solution $u_{0}^{-} \in N_{\lambda}^{-}$. Since $N_{\lambda}^{-} \cap N_{\lambda}^{+}=\emptyset$, then $u_{0}^{-}$and $u_{0}^{+}$are distinct.

\section{Conflict of Interests}

The authors declare that there is no conflict of interests regarding the publication of this paper.

\section{References}

[1] A. Ambrosetti, H. Brezis, and G. Cerami, "Combined effects of concave and convex nonlinearities in some elliptic problems," Journal of Functional Analysis, vol. 122, no. 2, pp. 519-543, 1994.

[2] C. O. Alves and Y. H. Ding, "Multiplicity of positive solutions to a $p$-Laplacian equation involving critical nonlinearity," Journal of Mathematical Analysis and Applications, vol. 279, no. 2, pp. 508-521, 2003.

[3] H. Brézis and E. Lieb, "A relation between pointwise convergence of functions and convergence of functionals," Proceedings of the American Mathematical Society, vol. 88, no. 3, pp. 486490, 1983.

[4] C.-M. Chu and C.-L. Tang, "Existence and multiplicity of positive solutions for semilinear elliptic systems with Sobolev critical exponents," Nonlinear Analysis: Theory, Methods \& Applications, vol. 71, no. 11, pp. 5118-5130, 2009.

[5] D. Lü, "Multiple solutions for $p$-Laplacian systems with critical homogeneous nonlinearity," Boundary Value Problems, vol. 2012, article 27, 2012. 
[6] G. A. Afrouzi and M. Alizadeh, "Positive solutions for a class of p-Laplacian systems with sign-changing weight," International Journal of Mathematical Analysis, vol. 1, no. 17-20, pp. 951-956, 2007.

[7] S. H. Rasouli and G. A. Afrouzi, "The Nehari manifold for a class of concave-convex elliptic systems involving the $p$-Laplacian and nonlinear boundary condition," Nonlinear Analysis: Theory, Methods \& Applications, vol. 73, no. 10, pp. 3390-3401, 2010.

[8] H. Brézis and L. Nirenberg, "Positive solutions of nonlinear elliptic equations involving critical Sobolev exponents," Communications on Pure and Applied Mathematics, vol. 36, no. 4, pp. 437-477, 1983.

[9] H. Yin, "Existence results for classes of quasilinear elliptic systems with sign-changing weight," International Journal of Nonlinear Science, vol. 10, no. 1, pp. 53-60, 2010.

[10] K. J. Brown and Y. Zhang, "The Nehari manifold for a semilinear elliptic equation with a sign-changing weight function," Journal of Differential Equations, vol. 193, no. 2, pp. 481-499, 2003.

[11] N. Nyamoradi, "The Nehari manifold for a Navier boundary value problem involving the p-biharmonic," Iranian Journal of Science and Technology, vol. 35, no. 2, pp. 149-155, 2011.

[12] M. Willem, Minimax Theorems, Progress in Nonlinear Differential Equations and their Applications, 24, Birkhäuser, Boston, Mass, USA, 1996.

[13] X.-F. Ke and C.-L. Tang, "Existence of solutions for a class of noncooperative elliptic systems," Journal of Mathematical Analysis and Applications, vol. 370, no. 1, pp. 18-29, 2010.

[14] T.-F. Wu, "On semilinear elliptic equations involving concaveconvex nonlinearities and sign-changing weight function," Journal of Mathematical Analysis and Applications, vol. 318, no. 1, pp. 253-270, 2006. 


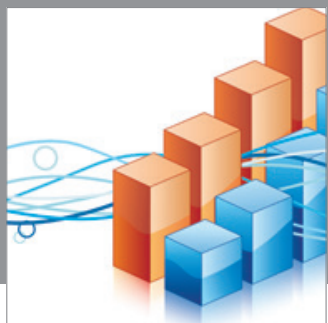

Advances in

Operations Research

mansans

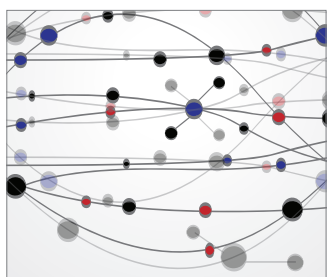

The Scientific World Journal
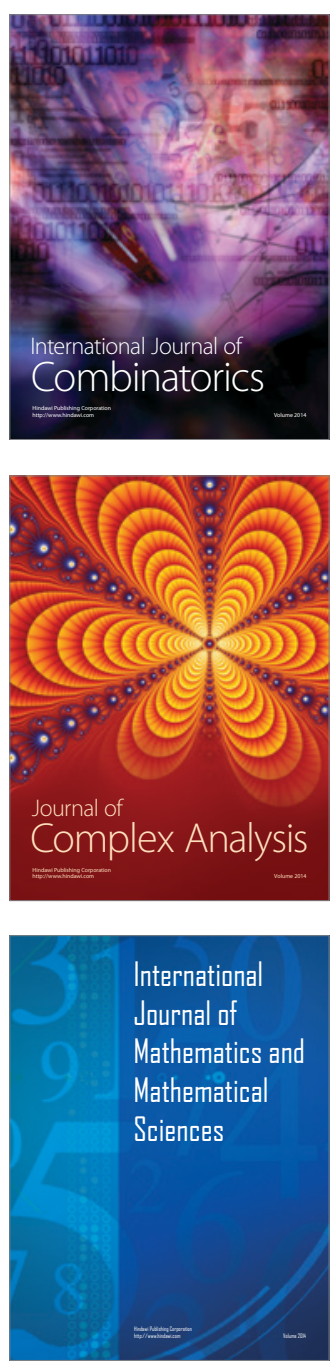
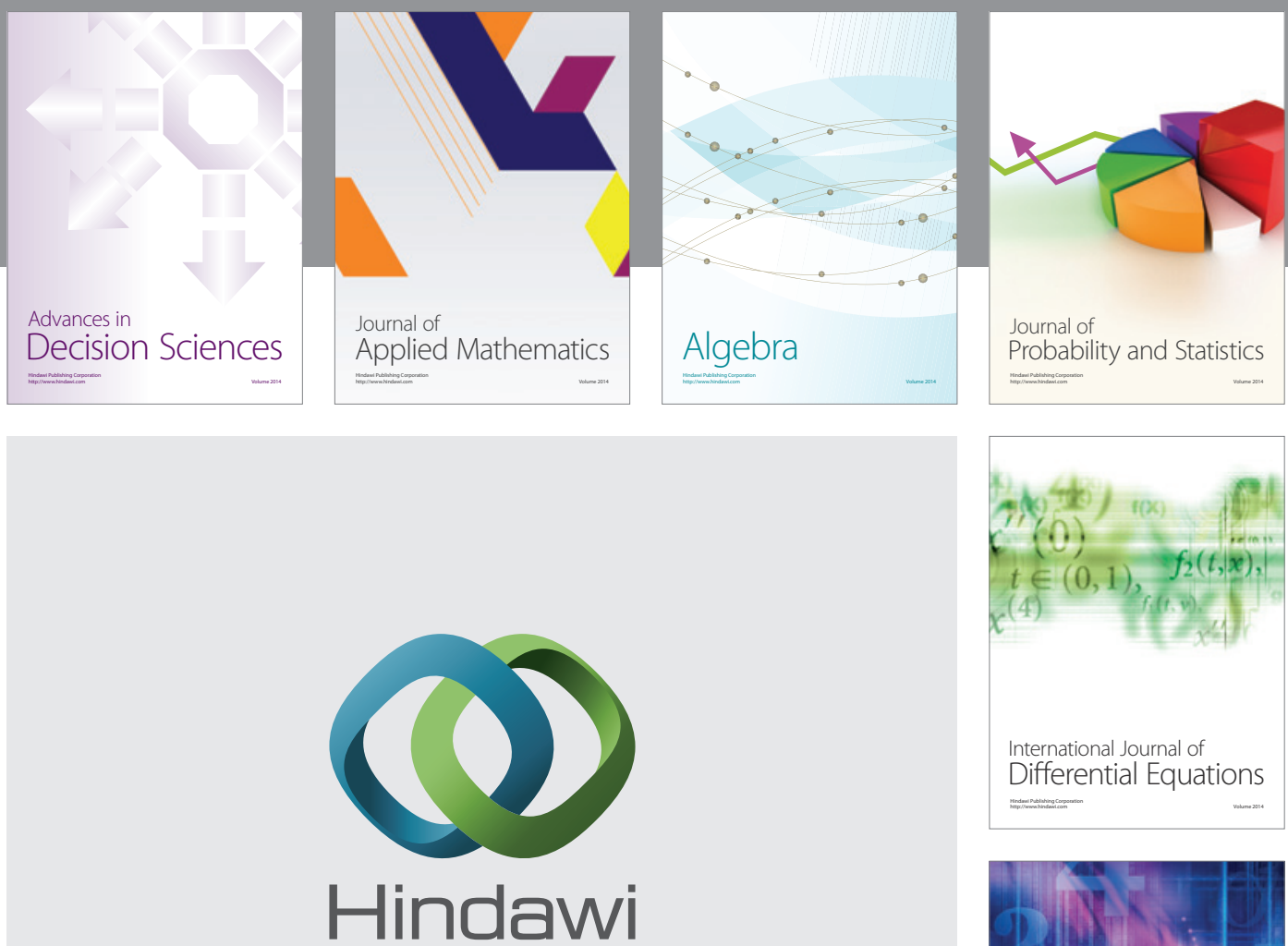

Submit your manuscripts at http://www.hindawi.com
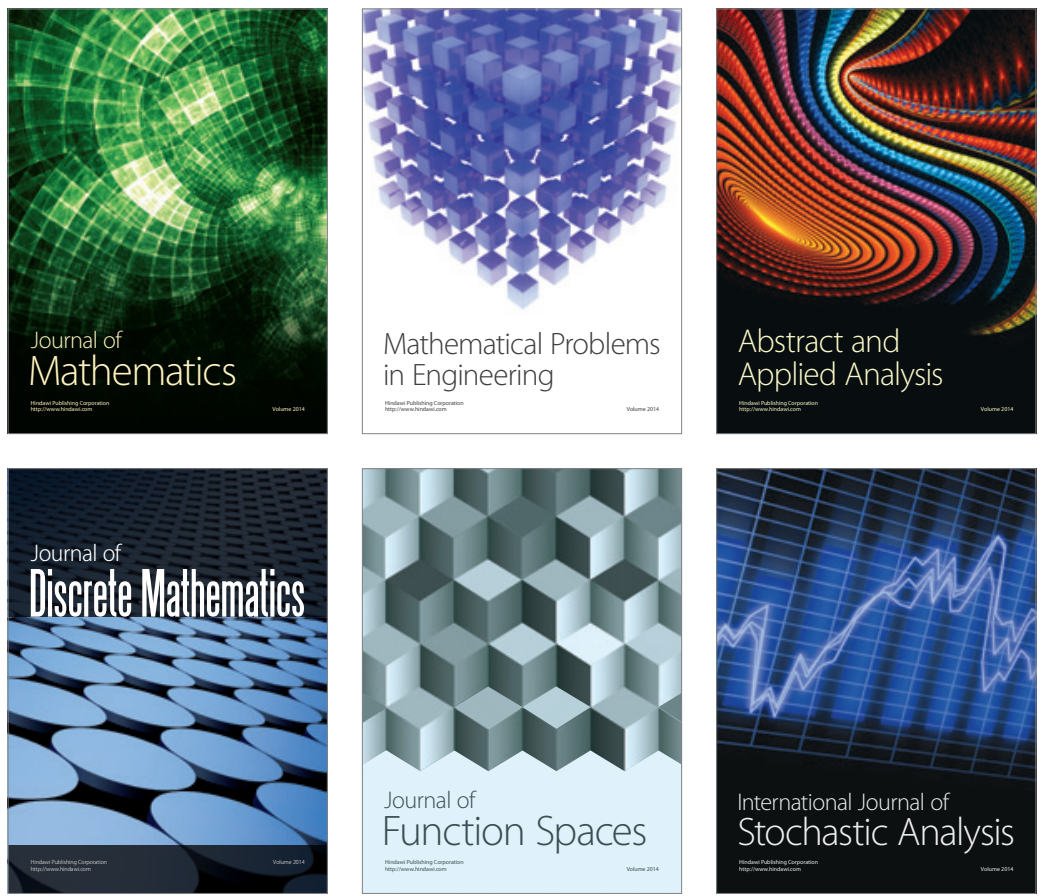

Journal of

Function Spaces

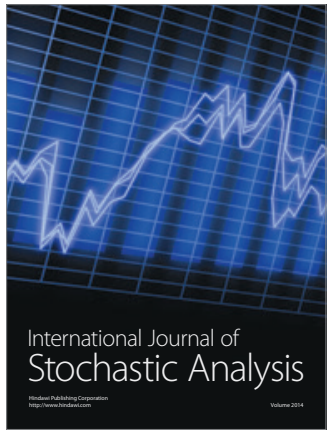

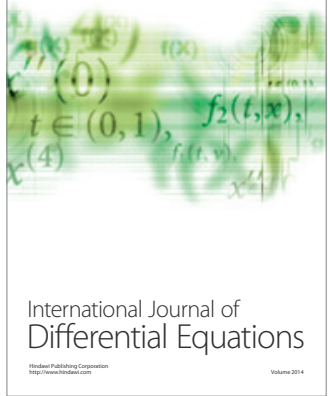
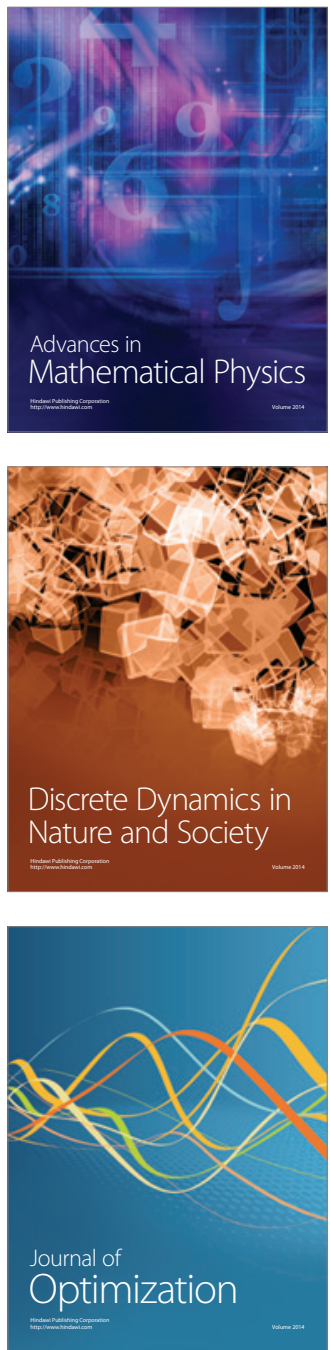